\title{
Evaluation of the Genetic Risk of Hypertension-Related Diseases
}

\author{
Hironori Nakagami, MD, PhD
}

$\mathbf{G}$ enetic predisposition is a well-known risk factor in life-style related diseases such as hypertension, myocardial infarction and diabetes mellitus. More than 20 years ago, the frequency of a homozygous deletion genotype (DD) of the angiotensin-converting enzyme (ACE) gene was associated with myocardial infarction. ${ }^{1}$ The impact of that study caused us to recognize the importance of genetic information, not only for inherited diseases but also for lifestylerelated diseases. Most scientists support the advent of tailor-made medicine by genetic analysis, ${ }^{2}$ and indeed, the ACE genotype has been intensively studied in association with hypertension and hypertension-related diseases. ${ }^{3-6}$ In this case, genotype determination may be useful in the assessment of coronary artery disease (CAD) risk. In addition, several other genotypes of functional genes (ie, angiotensinogen, ${ }^{7}$ endothelial nitric oxide synthase $[\mathrm{eNOS}]^{8}$ ) have been reported to be associated with hypertension; however, the contribution of each single-nucleotide pleomorphism (SNP) was not very powerful in its association with hypertension or hypertension-related diseases. With the technical progress in genetic analyses, large-scale genomewide association studies (GWAS) have been successful in identifying genes that contribute to common diseases and phenotypes. A GWAS of hypertension-related phenotypes in a Japanese population has been conducted, and the ATPase, $\mathrm{Ca}^{2+}$ transporting, plasma membrane 1 gene (ATP2B1) was successfully identified as a gene responsible for hypertension not only in Japanese patients but also Caucasians. ${ }^{9}$

\section{Article p 830}

In this issue of the Journal, Dr Kato's group, a leading group in this field, demonstrate that a genetic risk variant associated

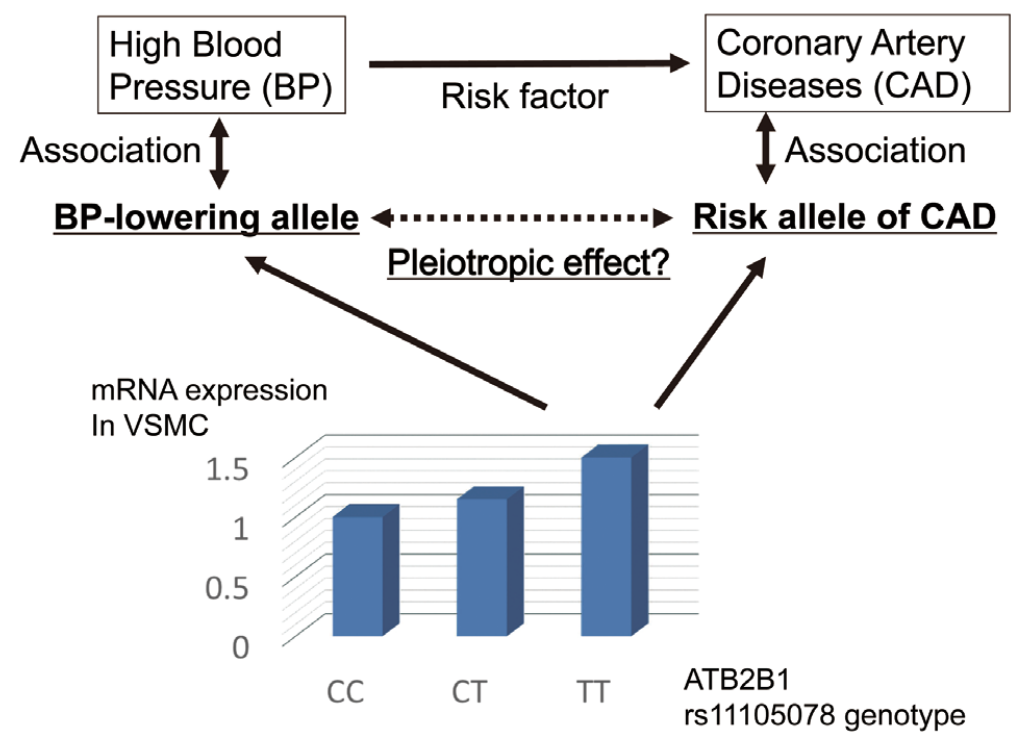

Figure. ATP2B1 mRNA expression levels in umbilical artery smooth muscle cells increased in BP-lowering ATP2B genotypes. Although hypertension is usually related to an increased risk of CAD, SNPs on the BP-lowering allele of ATP2B1 were associated with increased risk of CAD. Are these observations "pleiotropic" effects? BP, blood pressure; CAD, coronary artery disease; SNP, single-nucleotide pleomorphism.

The opinions expressed in this article are not necessarily those of the editors or of the Japanese Circulation Society.

Received February 15, 2015; accepted February 16, 2015; released online February 25, 2015

Division of Vascular Medicine and Epigenetics, Osaka University United Graduate School of Child Development, Suita, Japan

Mailing address: Hironori Nakagami, MD, PhD, Division of Vascular Medicine and Epigenetics, Osaka University United Graduate School

of Child Development, 2-2 Yamada-oka, Suita 565-0871, Japan. E-mail: nakagami@gts.med.osaka-u.ac.jp

ISSN-1346-9843 doi:10.1253/circj.CJ-15-0178

All rights are reserved to the Japanese Circulation Society. For permissions, please e-mail: cj@j-circ.or.jp 
with increased blood pressure (BP) also correlated with the risk of CAD in East Asians. ${ }^{10}$ These authors selected 9 SNPs confirmed to be associated with BP in East Asians. In the combined sample (6,522 cases and 11,263 controls), significant CAD associations were found with 5 SNPs. Among these, SNPs on the BP-lowering allele of ATP2B1 were associated with increased risk of CAD. Although hypertension is usually related to an increased risk of $\mathrm{CAD}$, this result suggests that the association between the ATP2B1 SNP and CAD might be through a non-BP-mediated mechanism (Figure).

Tabara et al analyzed the association of ATP2B1 SNPs with hypertension in a Japanese patient population ${ }^{9}$ and showed a significant association between hypertension and an ATP2B1 SNP (rs111053781; odds ratio 1.31 [95\% confidence interval: 1.21-1.42]; $\left.\mathrm{P}=4.1 \times 10^{-11}\right)$. In addition, ATP2B1 mRNA expression levels in umbilical artery smooth muscle cells were significantly different among individuals with the rs11105378 genotypes (Figure). The ATP2B1 gene removes bivalent calcium ions from eukaryotic cells against very large concentration gradients and plays a critical role in intracellular calcium homeostasis. It has been reported that the inhibition of ATP2B1 by a selective inhibitor showed endothelium-dependent relaxation of rat aorta by increasing cytosolic $\mathrm{Ca}^{2+}$ concentrations and consequent activation of eNOS, ${ }^{11}$ which may be related to hypertension. Thus, the question becomes why is the BPlowering allele of ATP2B1 associated with increased risk of CAD?

Takeuchi et al $^{10}$ discussed the discrepancy of an increased risk of CAD not always being related to a BP locus. For example, at 12q24.13 near ALDH2, the SNP alleles associated with elevated BP correlate with a reduced risk of CAD. ${ }^{12}$ Are these observations "pleiotropic" effects; that is, the deleterious effects of the variant (at 12q24.13) on BP are balanced by its protective effects on high-density lipoprotein and low-density lipoprotein cholesterol levels. In terms of the ATP2B1 phenotype, the authors ${ }^{12}$ proposed 2 hypotheses. One is that at a given locus there are multiple genes and alleles that participate in the regulation of multiple, independent traits through diverse mechanisms. These multiple variants might have arisen at different times during evolution, with each affecting CAD risk phenotypes independently, while the separate alleles with balancing effects have been fixed on a haplotype. The other hypothesis speculates that a single causal variant accounts for the observed associations with multiple CAD risk phenotypes in a pleiotropic manner. Although the exact mechanism behind this genetic analysis is not known, the potential mechanism behind ATP2B1 SNPs is interesting in the process of atherosclerosis or CAD.

Moreover, the G allele of rs12413409 (CNNM2; cyclin M2) was associated with reduced body mass index, whereas it had been associated with an increased risk of CAD and elevated $\mathrm{BP}$ in other studies. ${ }^{9} \mathrm{CNNM} 2$ could be part of the longsought basolateral $\mathrm{Mg}^{2+}$ extruder at the renal distal convoluted tubule, or its regulator. ${ }^{13}$ CNNM2 increases cellular $\mathrm{Mg}^{2+}$ uptake in HEK293 cells. In addition, patients from 5 families with mutations in the CNNM2 gene suffered from mental retardation, seizures, and hypomagnesemia. ${ }^{14}$ Although CNNM2 is fundamental for brain development, neurological functioning and $\mathrm{Mg}^{2+}$ homeostasis, it can also be associated with the development of hypertension or CAD through direct or indirect pathways.

In summary, the genetic analysis presented here suggests that a substantial number of genetic variants associated with $\mathrm{BP}$ are inversely associated with the risk of CAD. A metaanalysis of pharmacological studies showed that a mean BP decrease of approximately $1 \mathrm{mmHg}$ for SBP may reduce CAD risk by only $2.3 \% .{ }^{15}$ To further evaluate the risk of CAD, the combined effects of genetic information, clinical observations, biomarkers, patient characteristics, and so-called patient heterogeneity, should be calculated when considering the potential risk for each patient. We hope that this heterogeneity will be appreciated as tailor-made medicine evolves.

\section{Disclosures}

Conflict of Interest: The Division of Vascular Medicine and Epigenetics is endowed by Bayer.

\section{References}

1. Cambien F, Poirier O, Lecerf L, Evans A, Cambou JP, Arveiler D, et al. Deletion polymorphism in the gene for angiotensin-converting enzyme is a potent risk factor for myocardial infarction. Nature 1992; 359: 641-644.

2. Shimizu W. Update of diagnosis and management of inherited cardiac arrhythmias. Circ J 2013; 77: 2867-2872.

3. Marian AJ, Yu QT, Workman R, Greve G, Roberts R. Angiotensinconverting enzyme polymorphism in hypertrophic cardiomyopathy and sudden cardiac death. Lancet 1993; 342: 1085-1086.

4. Agerholm-Larsen B, Nordestgaard BG, Steffensen R, Sorensen TI, Jensen G, Tybjaerg-Hansen A. ACE gene polymorphism: Ischemic heart disease and longevity in 10,150 individuals: A case-referent and retrospective cohort study based on the Copenhagen City Heart Study. Circulation 1997; 95: 2358-2367.

5. Lindpaintner K, Pfeffer MA, Kreutz R, Stampfer MJ, Grodstein F, LaMotte F, et al. A prospective evaluation of an angiotensin-converting-enzyme gene polymorphism and the risk of ischemic heart disease. N Engl J Med 1995; 332: 706-711.

6. Funada A, Konno T, Fujino N, Muramoto A, Hayashi K, Tsubokawa $\mathrm{T}$, et al. Impact of renin-angiotensin system polymorphisms on development of systolic dysfunction in hypertrophic cardiomyopathy: Evidence from a study of genotyped patients. Circ J 2010; 74: 2674-2680.

7. Katsuya T, Koike G, Yee TW, Sharpe N, Jackson R, Norton R, et al. Association of angiotensinogen gene T235 variant with increased risk of coronary heart disease. Lancet $1995 ; 345$ : 1600-1603.

8. Wang XL, Sim AS, Badenhop RF, McCredie RM, Wilcken DE. A smoking-dependent risk of coronary artery disease associated with a polymorphism of the endothelial nitric oxide synthase gene. Nat Med 1996; 2: $41-45$.

9. Tabara Y, Kohara K, Kita Y, Hirawa N, Katsuya T, Ohkubo T, et al. Common variants in the ATP2B1 gene are associated with susceptibility to hypertension: The Japanese Millennium Genome Project. Hypertension 2010; 56: $973-980$

10. Takeuchi F, Isono M, Yamamoto K, Yokota M, Akiyama K, Katsuya $\mathrm{T}$, et al. Heterogeneous effects of association between blood pressure loci and coronary artery disease in East Asian individuals. Circ $J$ 2015; 79: 830-838.

11. Chaudhary J, Walia M, Matharu J, Escher E, Grover AK. Caloxin: A novel plasma membrane $\mathrm{Ca}^{2+}$ pump inhibitor. Am J Physiol Cell Physiol 2001; 280: C1027-C1030.

12. Preuss M, König IR, Thompson JR, Erdmann J, Absher D, Assimes TL, et al. Design of the Coronary ARtery DIsease Genome-Wide Replication And Meta-Analysis (CARDIoGRAM) Study: A genomewide association meta-analysis involving more than 22000 cases and 60000 controls. Circ Cardiovasc Genet 2010; 3: 475-483.

13. Hirata Y, Funato Y, Takano Y, Miki H. $\mathrm{Mg}^{2+}$-dependent interactions of ATP with the cystathionine- $\beta$-synthase (CBS) domains of a magnesium transporter. J Biol Chem 2014; 289: 14731-14739.

14. Arjona FJ, de Baaij JH, Schlingmann KP, Lameris AL, van Wijk E, Flik G, et al. CNNM2 mutations cause impaired brain development and seizures in patients with hypomagnesemia. PLoS Genet 2014; 10: e1004267, doi:10.1371/journal.pgen.1004267.

15. Staessen JA, Gasowski J, Wang JG, Thijs L, Den Hond E, Boissel $\mathrm{JP}$, et al. Risks of untreated and treated isolated systolic hypertension in the elderly: Meta-analysis of outcome trials. Lancet 2000; 355: 865-872. 